\title{
Les livres de rêve des savants
}

\section{Jacqueline Carroy}

Nuits savantes. Une histoire des rêves (1800-1945)

Paris, Éd. de l'École des hautes études en sciences sociales, coll. «En temps \& lieux », $2012,460 \mathrm{p}$.

Les chercheurs en sciences humaines et sociales, aujourd'hui, ne s'adonnent plus guère à une pratique qui, du début du $\mathrm{XIX}^{\mathrm{e}}$ siècle au milieu du $\mathrm{XX}^{\mathrm{e}}$ siècle, était relativement courante : la tenue d'un livre de rêves ou d'un «nocturnal» (selon le néologisme d'Antoine Charma) et l'utilisation des matériaux ainsi collectés dans des ouvrages ou articles à prétention scientifique. Comme le note Jacqueline Carroy, au terme d'une fascinante plongée dans l'intimité des «savants rêveurs » qui, de Charma à Maurice Halbwachs en passant par Alfred Maury, Joseph Delbœuf ou Sigmund Freud, s'efforcèrent de comprendre leurs propres productions oniriques (et les mécanismes généraux du rêve) en vue d'étayer des projets de connaissance et de théorisation de la spécificité des êtres humains parfois extrêmement ambitieux :

Actuellement, avec la diffusion de la psychanalyse et le développement des laboratoires et de la physiologie du sommeil, publier des récits oniriques bruts, avec un propos scientifique, qui plus est en son nom propre, est devenu quelque peu naïf. Les rêves à citer sont désormais, pour l'essentiel, anonymes, soit qu'ils servent d'exemples à des psychanalystes ou à des psychologues, soit qu'ils figurent dans des «banques de rêves » sur Internet. Seuls, ou presque, les écrivains, dans la mouvance du surréalisme et de la psychanalyse, signent désormais leurs songes (p. 411).

La situation est d'autant plus étrange que les chercheurs ont été largement incités, ces dernières années, à opérer un retour réflexif sur eux-mêmes. Or, si l'on en juge par les exercices d'ego-histoire ou d'auto-analyse - parfaitement maîtrisés - auxquels se sont livrés récemment quelques grands noms, le tabou du rêve a encore de beaux jours devant lui. Les spécialistes des sciences humaines et sociales, de toute évidence, ne notent plus leurs songes et ne sont plus prêts à sonder leurs désirs inavouables. En quoi ils se privent d'un instrument 
de connaissance d'eux-mêmes probablement irremplaçable. Consultant, vers le début des années 1940, ses «fiches de rêves » bien longtemps après qu'elles eurent été rédigées, Maurice Halbwachs fut ainsi impressionné par leur valeur documentaire autobiographique. Il en témoigna dans l'introduction d'un long article sur «Le rêve et le langage inconscient du sommeil » publié en 1946 (un an après sa mort au camp de Buchenwald) dans le Journal de psychologie normale et pathologique, article peu connu qu'exhume opportunément Carroy :

En relisant tous ces songes que j'ai notés, et l'interprétation que j'en ai donnée au réveil, et que j'ai aussi reproduite, que de circonstances de ma vie en cette période de vingt années je puis évoquer, que de personnages oubliés, d'événements auxquels je n'avais plus pensé, reparaissent soudain, comme si je relisais un journal où $\mathrm{j}$ 'aurais inscrit fidèlement tout le détail de ma vie quotidienne, non seulement les faits importants, mais des incidents secondaires et presque insignifiants, des pensées fugitives, des propos auxquels l'attention ne s'attache que sur le moment ! C'est tout cela qui constitue la matière d'où le rêve tire toutes ses créations (cit., p. 395).

Si le sociologue n'avait pas pris l'habitude de noter ses rêves, soit sous la forme de fiches «scientifiques» (hélas perdues), soit, plus rarement, dans son journal (heureusement conservé), on ignorerait, par exemple, à quel point la rivalité qu'il entretenait avec le psychologue Charles Blondel l'obséda tout au long de sa carrière. Dans l'un de ses rêves, consigné en 1935, il est en effet question de Blondel et apparaît, figure paternelle marmoréenne, une sorte de personnage double (Durkheim et Lévy-Bruhl); ce rêve, qu'évoque Carroy et qu'avait auparavant analysé Laurent Mucchielli ${ }^{1}$, trahit très probablement l'angoisse qui habitait Halbwachs de ne pas parvenir à triompher de son adversaire aux yeux de ses pères spirituels, en dépit de sa nomination à la Sorbonne. Une telle source ne nous offre pas seulement une illustration assez fascinante d'une situation concurrentielle que la lecture des textes et la reconstitution des positions sociales et intellectuelles permettent le plus souvent d'objectiver; elle nous donne aussi accès - c'est ce qui fait son prix - à tout un ensemble de sentiments et d'expériences vécues, généralement refoulés ou tenus sous le boisseau : jalousie, doutes, ambitions, mauvaise conscience, besoin irrépressible de reconnaissance, quête de l'approbation parentale, fantasme parricide ou fratricide, etc.

Sans doute ne faut-il pas demander aux rêves plus qu'ils ne peuvent offrir ; ils ne nous font pénétrer dans aucun territoire absolument inconnu et il est vain de chercher en eux le secret des créations humaines, contrairement à ce qu'avait pu laisser espérer le fameux rêve

\footnotetext{
${ }^{1}$ Voir L. Mucchielli, « Pour une psychologie collective : I’héritage durkheimien d'Halbwachs et sa rivalité avec Blondel durant l'entre-deux-guerres ", Revue d'histoire des sciences humaines, n 1, 1999, p. 110.
} 
de la sonate de Tartini ${ }^{2}$. Ce sont les relations de la prime enfance et, sur cette base, les expériences de la vie adulte telles qu'elles sont vécues dans des sociétés divisées en sousespaces gouvernés par des enjeux de reconnaissance immanents que les rêves permettent aujourd'hui le plus sûrement d'atteindre, pour peu que l'on veuille bien accepter - au croisement de ce que Freud a appelé, pour le meilleur et pour le pire, la «pulsion de vie » et la «pulsion de mort »-1'ambivalence structurelle objective des sentiments affectifs que l'on éprouve, à des degrés divers, envers autrui.

Pour l'historien des sciences humaines et sociales, les «livres de rêve » des savants présentent un double intérêt. Tout d'abord, ils aident à penser, hors des sentiers battus, l'enjeu central de ces sciences, à savoir la théorisation, fondée sur les faits, de l'entrecroisement individuellement structurant des propriétés «physio-psychologiques » universelles des êtres humains et des différents registres d'expériences relationnelles qu'ils subissent dans des cadres «familiaux » et « sociaux » préétablis ; ensuite, ils permettent de cerner la personnalité profonde des chercheurs confrontés à ce réquisit même. Historienne, spécialiste réputée de la psychologie du XIX ${ }^{\mathrm{e}}$ siècle, Jacqueline Carroy s'est donnée les moyens d'actionner cette clef, jetant ainsi une lumière inédite sur le développement des sciences humaines et sociales entre 1800 et 1945.

En s'intéressant aux philosophes, médecins, psychologues et « amateurs cultivés, souvent pourvus de titres académiques » (p. 14), qui s'attachèrent durant cette période à noter leurs rêves et, pour certains, à recueillir autour d'eux autant de rêves «fiables » que possible, Jacqueline Carroy s'attaque, en effet, à un genre de production scientifique mal connu : le «livre de rêve », qui fait exploser la frontière entre la vie publique du savant voué à la postérité et la vie privée du simple mortel (puisque «l'auteur y est à la fois un observateur scientifique et celui d'une individualité et d'un “tempérament" particuliers » [p. 17]).

La période qu'elle couvre est charnière, qui se caractérise notamment par la cristallisation d'un ensemble de concepts généraux interconnectés bornant l'horizon de la recherche et de la réflexion sur les êtres humains : «biologique », «physiologique »,

\footnotetext{
${ }^{2}$ Giuseppe Tartini (1692-1770), violoniste et compositeur vénitien, prétendait avoir transcrit à son réveil une sonate admirablement exécutée pendant son sommeil par le diable. Le thème fut repris par Moreau de la Sarthe en 1820, déformé, exagéré durant tout le XIX ${ }^{\mathrm{e}}$ siècle, et finalement restauré dans son intégrité, expliqué scientifiquement et dépouillé de tout merveilleux, notamment par Freud, le diable devenant, pour lui, "l'assistance fournie par d'obscures puissances des profondeurs du psychisme » (cité p. 226).
} 
«psychologique », «mental », « sociologique», «social», etc. Et effectivement, qu'il s'agisse de repérer dans les rêves l'influence du «physiologique » (ou du «physique ») sur le «mental» (ou sur le «moral»), d'illustrer la marche autonome du «psychique» ou de mesurer l'impact du «social» sous toutes ses formes (des rapports parents/enfant circonscrits par les psychanalystes au triangle œdipien - aux rapports gouvernants/gouvernés en passant par les relations professionnelles) sur le psychisme individuel, c'est bien une connaissance des êtres humains exclusivement fondée sur l'examen des faits qui est visée. En quoi, on le voit, les «savants rêveurs » portent remarquablement témoignage d'un nouveau régime conceptuel dont la mise en place participa de l'institutionnalisation des disciplines des sciences humaines et sociales, en rupture avec les traditions de pensée d'ordres religieux ou métaphysique.

Ce régime conceptuel est dominé, pour l'essentiel, par la problématique des rapports individu/société (qui autorise à prendre congé des schèmes de pensée dualistes tout en leur ouvrant des opportunités infinies de reconversion) et par la question de l'articulation complexe des phénomènes dits «biologiques » «psychologiques » et « sociaux » (qui permet d'accueillir de manière plus satisfaisante les résultats toujours provisoires des enquêtes empiriques menées, par nécessité, sur des fronts étroitement délimités) ${ }^{3}$. Ainsi, si l'étude des rêves fut d'abord surdéterminée par le problème de l'âme et du corps, elle offrira ensuite la possibilité de le dépasser à la faveur de la spécification d'un «psychique » dont le fonctionnement propre - irréductible au «physiologique» et au «social» bien qu'inconcevable sans eux - se révélera avec éclat dans ce qui commencera à être compris par des observateurs s'astreignant à noter méthodiquement leurs songes comme le travail même du rêve.

Cette évolution atteste peut-être l'existence d'une sorte de ligne de progrès scientifique. Jacqueline Carroy attire l'attention, à juste titre, sur les transformations du contexte institutionnel et sur les propriétés des acteurs concernés. Ainsi, en France, la philosophie spiritualiste et éclectique de Victor Cousin, enseignée dans les lycées, tenait le haut du pavé dans les années 1830-1860. Ceux qui se disaient psychologues et plaidaient pour le caractère sui generis des faits psychiques étaient d'abord et avant tout des philosophes qui croyaient, ou feignaient de croire pour des raisons politiques, en l'immortalité de l'âme, tout en étant parfois en butte à l'hostilité du clergé catholique; le «psychique», selon eux, était

\footnotetext{
${ }^{3}$ On décrit le déploiement de ce régime conceptuel dans un livre en cours de préparation. On a tenté d'analyser sa présence et sa mise en forme spécifique dans l'œuvre de Norbert Elias. Voir M. Joly, Devenir Norbert Elias. Histoire croisée d'un processus de reconnaissance scientifique : la réception française, Paris, Fayard, coll. « Histoire de la pensée », 2012.
} 
indissociable des deux facultés maîtresses : la volonté et la raison. Face à eux, les médecins, et notamment les aliénistes, défendaient le primat du «physiologique »; ils soulignaient l'importance du cerveau et se bornaient à constater dans les rêves produits par des sensations externes ou internes l'action du «physique» sur le «moral». Beaucoup d'entre eux cherchaient néanmoins des accommodements avec le spiritualisme. Ce sont ces deux catégories de savants qui, au départ, pouvaient parler avec autorité des rêves. Ils multipliaient, pour mieux les apprivoiser ou les circonscrire, les rapprochements avec d'autres phénomènes (la folie, le somnambulisme, etc.).

L'entrée en scène de deux acteurs qui n'étaient ni philosophes ni médecins, mais dont la légitimité reposait exclusivement sur l'investigation de leurs propres rêves (et de ceux de personnes de confiance), Alfred Maury et Léon d'Hervey de Saint-Denys, tous deux sinologues (plus ou moins amateurs) et professeurs au Collège de France, contribua à changer la donne. L'establishment spiritualiste, de toute façon, s'était montré assez malheureux dans ses tentatives d'encadrement de l'étude des rêves. Déjà, Charma, l'un des premiers savants à exciper de ses productions oniriques et à en proposer une théorie originale, spiritualiste conséquent qui avait une claire conscience de la limite séparant la psychologie de la physiologie, s'était éloigné peu à peu de l'orthodoxie cousinienne en mettant l'accent, « sous la dictée du rêve » (cit., p. 36), sur les profondes transformations nocturnes des facultés de l'âme ; élu professeur à la faculté des lettres de Caen en 1830 à l'âge de vingt-neuf ans et resté résolument «provincial» jusqu'à sa mort, il volait un peu de ses propres ailes. Quant au concours ouvert par l'Académie des sciences morales et politiques en 1851, à l'instigation de Cousin, invitant sans ambiguïté les candidats à privilégier les analogies avec le somnambulisme et à magnifier le rôle immuable des facultés de l'âme- et en premier lieu de la volonté-, il ne donna pas les résultats escomptés. Les personnalités les plus compétentes s'abstinrent d'y participer, les adeptes du magnétisme animal l'utilisèrent comme une tribune pour défendre leurs idées, et le vainqueur, un spiritualiste bon teint, Albert Lemoine, proposa une synthèse si prudente qu'elle ne convainquit personne, pas même le rapporteur de l'Académie.

Si Hervey de Saint-Denys, auteur en 1867 de Les rêves et les moyens de les diriger, se contenta de transmettre une sorte d' «art de bien rêver»(p. 117), Maury, lui, caressait l'ambition d'une théorie générale non pas seulement des rêves, mais de la psychologie humaine dans son entier. Carroy n'hésite pas à le comparer à Freud. Et c'est un fait que Le sommeil et les rêves, publié en 1861, dont l'édition revue et augmentée de 1878 eut beaucoup d'influence, constituait jusqu'à la Traumdeutung l'ouvrage de référence sur les rêves, tant sur 
un plan théorique que sur un plan documentaire. Érudit touche-à-tout, antiquaire, « compagnon de route des aliénistes » (p. 81), ami de Renan et de Flaubert, familier de Napoléon III, Maury voulait «édifier une science globale de l'homme qui mette l'accent sur “les rapports du moral et du physique', ainsi que sur les problèmes d'origine » (p. 82). Il visait, autrement dit, l'élaboration d'une psychologie « laïque » qui fût à la fois physiologique et historique. Ce qui en faisait un adversaire résolu du spiritualisme. La virtuosité avec laquelle il parvenait à interpréter ses propres rêves est patente, par exemple, dans l'analyse dont il gratifia en 1852 les membres de la Société médico-psychologique de trois rêves d'une même nuit, reliés entre eux par les mots «pèlerinage », «Pelletier » (nom d'un chimiste) et «pelle ». Mais le rêve qui lui valut une fortune durable est celui connu sous le nom de « rêve de la guillotine »: Maury rêve qu'il est condamné à être guillotiné par un tribunal révolutionnaire, se réveille en sueur, sentant choir sa tête, et remarque que la flèche de son lit s'est détachée et est «tombée sur [s]es vertèbres cervicales à la façon du couteau de la guillotine $»($ cit., p. 89). On voit, par ces exemples, qu'il savait aussi bien rendre compte de l'autonomie apparemment arbitraire du psychisme (manifeste dans les associations d'idées suivant librement un cours déroutant, mais néanmoins logiquement reconstituables au réveil, ou dans le surgissement imprévisible de penchants et passions réprimés) qu'analyser l'effet souvent déterminant de pures sensations externes. Sur ces deux points, il était éloigné d'Hervey de Saint-Denys, pour lequel l'esprit était non seulement en droit mais en capacité de contrôler le déroulement des associations d'idées.

Jusqu'à Freud, dans l'univers francophone, c'est Maury qui fit le plus d'émules et c'est à lui qu'il convenait de se mesurer. Il est même probable, indique Carroy, que Le sommeil et les rêves représentait, aux yeux de l'auteur de la Traumdeutung, un traité classique par rapport auquel il avait à se situer en priorité. Le projet freudien de synthèse et de refondation de l'interprétation des rêves présenterait en tout cas des analogies fortes avec les ambitions du « savant rêveur » français :

Comme Maury, Freud publie une somme ayant l'ambition d'une part de relier rêves et phénomènes pathologiques et d'autre part d'insérer les rêves dans le cadre d'une théorie générale du développement et de l'évolution. Comme lui, Freud propose à son lecteur une pratique et une méthode d'analyse des rêves dans un chapitre initial et, toujours comme lui, il multiplie les "spécimens de rêves avec leurs particularités, leurs détails, leurs indiscrétions, leurs mauvais jeux de mots", pour reprendre une lettre à Fliess du 6 août 1899 (p. 315). 
Freud était aussi critique à l'égard de l'automatisme psychologique de Maury et de son réalisme physiologiste que sensible à la perspicacité avec laquelle il avait su traduire le côté sombre des rêves - ces défis aux normes de la morale et du comportement consciemment éprouvées et appliquées - ; ce qui allait, bien entendu, dans le sens de la thèse principale qu'il entendait défendre : à savoir que le rêve constitue une satisfaction (plus ou moins) déguisée d'un désir (plus ou moins profondément) refoulé. De même, Gabriel Tarde, fasciné lui aussi par «l'imagination dépravée » (cit., p. 158) du dormeur et suivant l'exemple de Maury sur bien des points, ressentit le besoin de réhabiliter à l'encontre de celui-ci le romanesque du rêve.

Si la psychologie physiologique de Maury inspira des livres importants comme Les rêves. Physiologie et pathologie de Philippe Tissié, l'un des premiers à faire un usage thérapeutique des songes, elle fut aussi violemment critiquée, en particulier, nous apprend Carroy, dans les pages de la Revue philosophique de Théodule Ribot (lequel, par amitié pour Tarde, semble-t-il, accepta d'accueillir dans sa revue une sorte de poète d'avant-garde qui prétendait révolutionner la manière d'aborder les rêves). Hervey de Saint-Denys, avant de prendre ses distances avec l'étude des rêves, était si obnubilé par la rivalité objective qui le liait à Maury qu'il affirmait avoir interprété quelques-uns de ses songes au cours même de leur déroulement comme autant de désaveux des théories de son concurrent. Il est assez évident que cette rivalité souterraine ainsi que, par la suite, les apports de personnalités aussi diverses que le psychologue liégeois Delbœuf (qu'aucune «énigme» du rêve ne faisait reculer et qui aimait à «faire surgir tout un monde à partir d'un exemple initial » [p. 195]) ou les oubliés Victor Egger, Marcel Foucault et Yves Delage ont concouru, à partir de l'examen des rêves, à affiner l'armature du régime conceptuel des sciences humaines et sociales, le «physiologique» étant en l'occurrence de moins en moins envisagé sur un mode «autosuffisant» (avec, notamment, l'accent mis sur les sources profondes du «désir», comme chez Tarde), et mieux identifié par ses lois propres; le «psychique » étant compris indépendamment des présupposés de la philosophie spiritualiste et en rapport avec l'enfance ; et le «social», consciemment appréhendé à travers la révélation nocturne des relations réellement investies par le sujet. Mais, outre la consolidation et la sophistication progressives d'un cadre de pensée tridimensionnel («physio-psycho-sociologique »), l'enquête de Jacqueline Carroy atteste aussi la perpétuation de structures d'oppositions duelles (passé/présent, corps/esprit, etc.). L'historienne nous paraît ainsi avoir touché du doigt ce qui constitue la tension centrale du régime conceptuel des sciences humaines et sociales : la 
tension entre un schéma d'articulation tridimensionnel factuellement orienté et l'antinomie individu/société (sous toutes les formes qu' elle est susceptible de revêtir).

C'est dire que Nuits savantes invite à renouveler le regard porté sur l'histoire des sciences humaines et sociales. Les sources originales si fortement autobiographiques étudiées par l'auteure ainsi que la manière dont elle les a analysées donnent par ailleurs une idée du changement de perspective que pourrait produire une prise en compte systématique des expériences «existentielles » qui sont au principe des œuvres scientifiques.

Considérons le cas de Gabriel Tarde. On ne connaissait pas, jusqu'à tout récemment, ses rêves $^{4}$. Ceux-ci, notés entre 1870 et 1872 , peuvent mettre mal à l'aise tant leur auteur, longtemps handicapé par une maladie des yeux qui l'obligea à rester dans l'obscurité des mois durant et travaillé par ses échecs sentimentaux et ses pulsions sexuelles, était lui-même mal dans sa peau. Mais, en les examinant à la lumière des textes de fiction composés par Tarde à la même époque, Carroy suggère qu'ils sont riches d'enseignements, quant à la tendance tardienne d'un repli sur la sphère monadologique ou quant aux a priori idéologiques réactionnaires de l'auteur des Lois de l'imitation (soigneusement dissimulés par exemple par son ami Delbœuf).

Peut-on saisir ce que font de sa structure mentale innée et de son organisme l'ensemble des rapports humains vécus par le sujet? L'enchevêtrement processuel qu'il y aurait lieu, ainsi, de dégager et de dire est-il voué à échapper à l'intelligence et au langage ?

L'étude des rêves apporte peut-être une réponse. C'est du moins ce qu'ont voulu croire les «savants rêveurs » et les «rêveurs savants» ressuscités par Jacqueline Carroy. Il est d'autant plus fascinant de constater à quel point ils étaient prisonniers d'eux-mêmes (de leurs fantasmes, de leurs désirs, de leurs expériences fondatrices, des réponses données durant leur enfance à la manifestation de leurs pulsions de plaisir et d'auto-affirmation, etc.) ; à quel point ils restaient dépendants autant qu'ils ressentaient la nécessité de s'affranchir de certains schèmes de pensée, notamment ceux, prolongeant le vieil héritage de la métaphysique, légués par le spiritualisme. C'est peut-être pourquoi ils peuvent donner l'impression, a posteriori, d'avoir vu et dit l'essentiel sans le savoir ou sans en mesurer entièrement les conséquences, en dépit de leurs préjugés de tous ordres ou pis encore-(pour l'observateur naïf d'aujourd'hui)

\footnotetext{
${ }^{4}$ G. Tarde, Sur le sommeil ou plutôt sur les rêves. Et autres textes inédits, J. Carroy et L. Salmon (eds), Lausanne, BHMS, coll. « Sources en perspective », 2009.
} 
grâce à ces préjugés mêmes. Impression floue, assurément, puisqu'elle ne tient pas compte du fait qu'entre la prison intime et personnelle de l'enfance, et la prison des croyances et habitudes de pensée partagées avec d'autres, le rêve et son récit ont pu offrir une échappatoire - précisément en favorisant leur contact, leur ouverture l'une à l'autre. C'est le même Tarde qui, subissant l'ascendant de la philosophie spiritualiste et s'obstinant à répéter que l'on n'est ni « soi » ni chez « soi » dans les rêves, releva : «Tous nos sentiments se trahissent en songe ; toute notre vie s'y répète, notre cœur s'y révèle ; nous rêvons à nos amours, à nos inquiétudes, à nos espérances, à notre passé, plus souvent que nous ne pensons » (cit., p. 164).

Il y a là une dialectique libératrice dont on se demande bien comment elle pourrait opérer, subjectivement, autrement que par le récit du rêve. Le sommeil s'étudie aujourd'hui dans les laboratoires. Les rêves, eux, sont interprétés selon des modalités d'ordre «psychanalytique », contrôlées par l'analyste (à l'enseigne de la recherche privilégiée d'une présence des figures parentales et dans l'idée, sans doute raisonnable d'un point de vue clinique, que «le rêve est au service du sommeil $\left.{ }^{5} »\right)$, beaucoup moins selon des modalités d'ordre «sociologique » (à travers la mise au jour hiérarchisante de l'ensemble des relations constitutives du sujet), sans que l'on sache exactement pour quelles raisons la psychanalyse et la sociologie n'ont pas à penser les mêmes relations, ni à penser de la même façon les relations de ces relations avec le processus de maturation biologique des êtres humains et avec les mécanismes mal connus de la mémoire humaine ${ }^{6}$. Sauf exception, personne ne doute que le rêve témoigne d'un fonctionnement mental prédéterminé, ni qu'il traduise des caractéristiques explicables du cerveau humain. Et personne ne doute que sa matière soit constituée des diverses impressions et idées de la veille, ni qu'elle reflète l'état de nos relations passées et présentes aux autres (état dont on ne peut avoir pleinement conscience et dont la disposition est stratifiée et différenciée en tant qu'il y a des degrés dans les investissements affectifs auxquels on sacrifie sur la base des expériences de la prime enfance dont le souvenir luit comme un phare dans la brume). Mais seuls les récits de rêve ont permis, permettraient encore, à l'évidence, de faire tenir ensemble, concrètement, ces différentes dimensions habituellement opposées.

\footnotetext{
${ }^{5}$ Ch. Dejours, Le Corps, d'abord. Corps biologique, corps érotique et sens moral, Paris, Payot \& Rivages, coll. "Petite bibliothèque Payot ", 2003 (1 $1^{\text {re }}$ éd. 2001), p. 45.

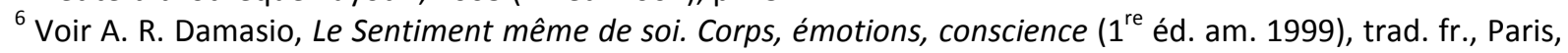
Odile Jacob, coll. « Sciences », 1999, p. 228-230.
} 
«Je ne rêve que pour atteindre la réalité ${ }^{7}$, fait dire à son ultime double la grande écrivaine brésilienne Clarice Lispector dans Un souffle de vie, ce « livre comme quand on dort et qu'on rêve intensément ${ }^{8} \gg$ dont le style et la composition évoquent de manière si troublante le télescopage désordonné des pensées et le rythme échevelé des associations d'idées et d'images à l'œuvre dans les rêves, et dont le titre même exprime probablement mieux que toutes les métaphores convenues la raison pour laquelle ceux-ci fascinent tant: ils sont des souffles de vie - vie de l'organisme, vie quotidienne dans ce qu'elle a de si précieusement banale, vie qui attire vers autrui, vie vécue au contact d'autrui.

Atteindre la réalité, saisir au vol des faits imaginaires réels qui nous parlent de nousmêmes et des relations qui nous ont faits tels que nous sommes et nous font être jour après jour, percer les mystères du cerveau, fonder une psychologie et/ou une physiologie, voire une nouvelle sociologie à la fois relationnelle et physio-psychologique ${ }^{9}$, tels étaient donc les objectifs poursuivis par les savants du $\mathrm{XIX}^{\mathrm{e}}$ siècle et des premières décennies du $\mathrm{XX}^{\mathrm{e}}$ siècle qui s'appliquèrent à scruter leurs rêves. Certains y passaient tant de temps qu'il leur arrivait de se souvenir de tel rêve précédent au cours de leur sommeil. Ils étaient des «amateurs » au sens noble du terme. Ils avaient la fraîcheur naïve des pionniers - et Jacqueline Carroy, si elle ne se prive pas, parfois, de faire sourire à leur propos, ne se laisse jamais aller à l'interprétation psychanalytique a posteriori (tentante, elle le reconnaît elle-même) ni à la condescendance : ses analyses sont fondamentalement frappées au coin de l'empathie et du respect des textes et des témoignages. Jusqu'à nous faire regretter que le genre scientifique pratiqué par ces «savants rêveurs» ait été dédaigné - «spécialisation» et « professionnalisation » obligent - avant même, peut-être, d'avoir pu porter tous ses fruits.

\footnotetext{
${ }^{7}$ Cl. Lispector, Un souffle de vie (1 ${ }^{\text {re }}$ éd. br. 1978), trad. fr., Paris, Des femmes, 1998, p. 69.

8 Ibid., p. 45.

${ }^{9}$ On peut remarquer qu'aucun des "savants rêveurs » de Carroy, hormis, peut-être, Maurice Halbwachs, trop tôt décédé, n'a pensé à formaliser une méthode aussi simple que celle consistant à analyser la manière dont les rôles sont distribués dans les rêves, en distinguant trois grandes catégories: les personnes connues personnellement (qui elles-mêmes se répartissent dans différentes catégories selon le degré de proximité affective : parents, conjoint, enfants, amis, relations professionnelles, connaissances, etc.) ; les personnages célèbres ou « icônes " (" personnalités " politiques ou artistiques, "figures » de tableaux, etc.) susceptibles de réveiller tel ou tel fantasme ou associées à tel ou tel désir non satisfait, ou à telle frustration ou telle blessure narcissique ; les personnes imaginaires créées à partir de ces deux catégories ou de notre moi dédoublé à la faveur du travail spécifique de figuration, de condensation et de déplacement effectué par le rêve. (On peut penser ici à Swaan, dans À la recherche du temps perdu, se reconnaissant sous les traits d'un jeune homme en fez et identifiant en la personne de Napoléon III l'amant de la personne qu'il croit ne plus aimer.) La méthode de décomposition des rêves en tableaux et en scènes, appliquée avec rigueur par Marcel Foucault dans son livre Le rêve. Études et observations (1906), se rapproche cependant de ce qu'aurait pu être une méthode de classification des figures humaines peuplant les rêves.
} 
Marc JOLY 